\title{
A Data-Driven Approach for the Prediction of Subcooled Boiling Heat Transfer
}

\author{
Jerol Soibam $^{1} \quad$ Ioanna Aslanidou $^{1} \quad$ Konstantinos Kyprianidis $^{1} \quad$ Rebei Bel Fdhila $^{1,2}$ \\ ${ }^{1}$ Mälardalen University, Västerås, Sweden, \{jerol.soibam, ioanna.aslanidou, \\ konstantinos.kyprianidis\} @mdh. se \\ ${ }^{2}$ Hitachi ABB Power Grids, Västerås, Sweden, rebei.bel_fdhila@hitachi-powergrids.com
}

\begin{abstract}
In subcooled flow boiling, heat transfer mechanism involves phase change between liquid phase to the vapour phase. During this phase change, a large amount of energy is transferred, and it is one of the most effective heat transfer methods. Subcooled boiling heat transfer is an attractive trend for industrial applications such as cooling electronic components, supercomputers, nuclear industry, etc. Due to its wide variety of applications for thermal management, there is an increasing demand for a faster and more accurate way of modelling.
\end{abstract}

In this work, a supervised deep neural network has been implemented to study the boiling heat transfer in subcooled flow boiling heat transfer. The proposed method considers the near local flow behaviour to predict wall temperature and void fraction of a sub-cooled mini-channel. The input of the network consists of pressure gradients, momentum convection, energy convection, turbulent viscosity, liquid and gas velocities, and surface information. The output of the model is based on the quantities of interest in a boiling system i.e. wall temperature and void fraction. The network is trained from the results obtained from numerical simulations, and the model is used to reproduce the quantities of interest for interpolation and extrapolation datasets. To create an agile and robust deep neural network model, state-of-the-art methods have been implemented in the network to avoid the overfitting issue of the model. The results obtained from the deep neural network model shows a good agreement with the numerical data, the model has a maximum relative error of $0.5 \%$ while predicting the temperature field, and for void fraction, it has approximately $5 \%$ relative error in interpolation data and a maximum $10 \%$ relative error for the extrapolation datasets.

Keywords: deep neural network (DNN), CFD, machine learning (ML), sub-cooled boiling, heat transfer.

\section{Introduction}

Subcooled flow boiling exists when the bulk liquid temperature remains below its saturation value, but the surface is hot enough for bubbles to form. Subcooled boiling is generally observed in micro-channel heat sinks, due to high heat transfer rates and maintaining relatively low wall temperature under high subcooled conditions. Due to the nature of high heat transfer mechanism in subcooled boiling, it plays a paramount role in enhancing the thermal efficiency of a system in industrial applications. However, the physical process that occurs during the subcooled boiling process is a complex phenomenon, and it remains a major challenge to predict the heat transfer behavior correctly in the boiling regime. One of the first attempts was made by Mohammed (1977), where they used multiple experimental data of different fluids to derive a correlation for low and high subcooled regions to predict the heat transfer coefficients. Kandlikar (1998) carried out an extensive review of the subcooled boiling heat transfer correlation, and he also introduced the region of void flow, where the convective heat transfer plays an important role due to void fraction. Yin et al.(2000) examined subcooled boiling heat transfer of R-134a in a horizontal annular duct, where they showed that increased subcooling had a huge effect in the bubble diameter. Through this work, they proposed an empirical correlation for boiling heat transfer coefficient and bubble departure diameter.

More recently, Multiphase Computational Fluid Dynamics (MCFD) models have been used for modeling boiling heat transfer and regarded as promising tools to understand the underlying physic in boiling flows. Jakobsen et al.(2005) reported that among the available modeling techniques, the Eulerian multi-fluid approach is most commonly used for bubble simulation. The Eulerian multi-fluid approach treats each phase as interpenetrating continua and relies on an ensemble averaging of the multiphase Navier-Stokes equations (Ishii, 1975). To solve the two-fluid model, a closure equation has to be introduced in the conservation equations for the individual phase, and the accuracy of the model highly depends on the closure equations (Besagni et al., 2018). These closure terms are combination of mechanistic models and empirical correlation, including nucleation site density, bubble departure diameter, bubble departure frequency, and heat flux. A comprehensive review on 
MCFD from Cheung et al.(2014) stated that there is no single combination of empirical correlations that can provide satisfactory predictions for all the quantities of interest for a variety of input conditions.

Machine learning (ML) algorithms provide an agile framework for specific problems, let it be medical data or fluid mechanics data. These algorithms can be used as an additional tools to optimize, understand the correlation that exist in data, speed up simulation and, better understand the underlying physics that occurs in fluid flow simulation or heat transfer problems. In recent years ML techniques are rapidly making an inroad towards fluid mechanics and heat transfer domain, and this is becoming possible due to high-performance computing and the advancement of experimental techniques. Ansari et al.(2020) successfully implemented Artificial Neural Network (ANN) for multiphase flow to predict the dynamic features including pressure, velocity, and the evolution of phase fraction. They claimed that their model was capable of predicting within few minutes under 10 $\%$ error. Alic et al.(2019) tested various ML algorithms (Genetic algorithms, ABC algorithms, Support vector machine (SVM), Decision Tree (DT), and Multi-Layer Perceptron (MLP)) to predict the heat flux of pool boiling heat transfer. According to their study SVM regression model gave the best prediction. Kumar et al.(2018) use ANN as an inverse model to estimate unknown heat flux in fin heat transfer with a maximum error of $0.41 \%$. They used surrogate data generated from Asymptotic CFD as an input to the ANN. Hobold and da Silva(2019) applied supervised and unsupervised ML techniques to study boiling heat transfer mechanisms, concerning bubble dynamics and bubble morphology on a wire. They demonstrated that the ANN-based model could quantify heat transfer using only direct and indirect visual information of the boiling phenomenon. Moreover, they used these models to infer heat flux in real-time using Raspberry Pi. Buist et al.(2019) used ML algorithms to learn the closure term for stratified multiphase flow in channels, from an unsteady high-fidelity simulation data. Then, the ML model was used to establish a functional relation between the two-fluid model and the closure terms, which served as the source term to the two-fluid model. Ma et al.(2015) used a neural network to fit Direct Numerical Simulation (DNS) data to develop closure relations for the average two-fluid equations. Once the network was trained it was used for different initial velocity and void fraction. They observed that the average equations with the neural network closure model were capable of reproducing the main aspect of the DNS results. The aforementioned, methods focus on addressing the heat transfer behavior in pool boiling, multiphase flow in a channel, or deriving a closure model with the aid of ML techniques. However, according to the authors best knowledge, there is no study related to sub-cooled boiling heat transfer in mini-channel using
Deep learning techniques to predict the void fraction and the temperature field.

This work focuses on the investigation of a Deep Neural Network (DNN) model to estimate the quantities of interest for subcooled boiling in a mini-channel. Data for training this model is obtained from CFD simulations and the data are then divided into training, validation, and testing datasets. The model used in this work is tailored to predict the quantities of interest for varying heat flux and inlet velocities. This model has 16 input features, and 2 output features, namely wall temperature and void fraction. Overall, this study shows that the DNN model can capture the physics and non-linear behaviors that exist in subcooled boiling heat transfer in mini-channel. Once the DNN model is trained and validated it is used to predict the QoIs for a new case for interpolation and extrapolation datasets, and it predicted with exceptional accuracy. From the predictive nature of the trained DNN model and its predictive speed, it opens up the possibility to use such a model for the design phase in thermal management for subcooled boiling systems.

\section{Numerical Method for data genera- tion}

The data used for training the Deep NN in this work are obtained from applying Eulerian two-fluid method to model the subcooled boiling flow in a mini channel. Numerical simulations are performed using open-source software OpenFOAM. The three conservation equation of incompressible Navier-Stokes equations are solved to generate the data. The mass conservation equation for each phase can be written as following:

$$
\frac{\partial \alpha_{k} \rho_{k}}{\partial t}+\nabla \cdot\left(\alpha_{k} \rho_{k} \mathbf{U}_{k}\right)=\Gamma_{k i}-\Gamma_{i k}
$$

where $k$ is the phases, $\alpha$ is the void fraction, $\rho$ is the phase density, $\mathbf{U}$ is the phase velocity and $\Gamma$ is the mass transfer rate per unit volume. The momentum conservation equation for each phase:

$$
\begin{array}{r}
\frac{\partial \alpha_{k} \rho_{k} \mathbf{U}_{k}}{\partial t}+\nabla \cdot\left(\alpha_{k} \rho_{k} \mathbf{U}_{k} \mathbf{U}_{k}\right)=-\alpha_{k} \nabla p+\mathbf{R}_{k}+\mathbf{M}_{k}+ \\
\alpha_{k} \rho_{k} \mathbf{g}+\left(\Gamma_{k i} \mathbf{U}_{i}-\Gamma_{k i} \mathbf{U}_{k}\right)
\end{array}
$$

where $\nabla p$ is the pressure gradient, $\mathbf{R}$ is the combined turbulent and laminar stress term, calculated based on the Reynolds analogy, $\mathbf{g}$ is the gravitational acceleration, and $\mathbf{M}$ is the interfacial momentum transfer which accounts for the drag forces. The energy transport equation is written in terms of specific energy $h$ for each phase $k$ as following:

$$
\begin{aligned}
\frac{\partial \alpha_{k} \rho_{k} h_{k}}{\partial t}+\nabla \cdot\left(\alpha_{k} \rho_{k} \mathbf{U}_{k} h_{k}\right)= & \alpha_{k} \frac{D p}{D t}+\nabla\left(\alpha_{k} D_{t, k}^{e f f} \nabla h_{k}\right) \\
& +\Gamma_{k i} h_{i}-\Gamma_{i k} h_{k}+Q_{\text {wall }, k}
\end{aligned}
$$


The standard $k-\varepsilon$ turbulence model is used to account for turbulence dispersed flow in the vapor phase. To account for the turbulence flow behavior of the continuous phase, the Lahey $k-\varepsilon$ (Lahey Jr, 2005) turbulence model is used for the liquid phase. The $k-\varepsilon$ turbulence model is adopted since it is valid for fully turbulent flows and computationally inexpensive also due its robust nature for pipe flows. The boiling model used here is based on the well known RPI model (Kurul, 1991), where the total heat flux $q_{w}$ on the wall is divided in three components, evaporation heat flux $q_{w, e}$, forced convection heat flux $q_{w, c}$ and quenching heat flux $q_{w, q}$. The total applied heat flux can be written as follows:

$$
q_{w}=q_{w, e}+q_{w, c}+q_{w, q}
$$

For this simulation active nucleation site density is define on the surface where total heat flux is applied as per the correlation (Benjamin and Balakrishnan, 1997):

$$
N_{a}=218 \operatorname{Pr}_{l}^{1.63} \Delta T_{\text {sup }}^{3} \gamma^{-1} \theta^{-0.4}
$$

where $\operatorname{Pr}_{l}$ is the liquid Prandtl number, $\Delta T_{\text {sup }}$ is the wall superheat, $\gamma$ is a coefficient taking into account the liquid and heated surface thermophysical properties and $\theta$ is a coefficient taking into account the heated surface roughness and the system pressure.

The bubble departure diameter is calculated based on the semi-empirical model of Ünal (Ünal, 1976), given as following:

$$
d_{d e p}=\frac{2.4210^{-5} p^{0.709} a}{\sqrt{b \phi}}
$$

where $a$ and $b$ are the model coefficients, taking into account the working fluid and the heated surface thermophysical properties, and $\phi$ is a parameter controlled by the local flow velocity.

The closure term for the bubble departure frequency is calculated according to the mechanistic model of Brooks and Hibiki (Brooks and Hibiki, 2015) as:

$$
f_{\text {dep }}=\frac{C_{f d} \mathrm{Ja}_{w}^{0.82} N_{T}^{-1.46} \rho^{*-0.93} \operatorname{Pr}_{\text {sat }}^{2.36}}{d_{d e p}^{2}}
$$

where $C_{f d}$ is the model coefficient depending on the size of the channel where boiling occurs, $N_{T}$ is a dimensionless temperature, $\rho^{*}$ is a dimensionless density ratio, $\mathrm{Ja}_{w}$ is a modified Jacob number and $\operatorname{Pr}_{s a t}$ is the liquid Prandtl number evaluated at the corresponding saturation temperature. With the initial and boundary conditions specified along with closure terms, the solver is able to predict the boiling heat transfer accurately and data are generated.

\subsection{Data Extraction}

The 2D computational domain of the mini-channel used for simulation is shown in the Figure 1. The channel has a total height of $0.6 \mathrm{~m}$ along the $\mathrm{y}$-axis and $0.003 \mathrm{~m}$ along the x-axis. To avoid the influences of the boundary, nondeveloped flow, and to account, only the surface where

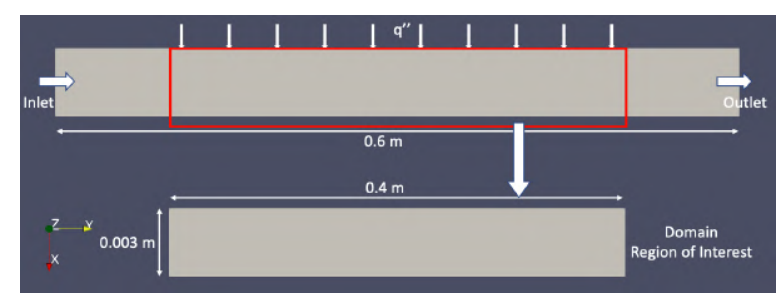

Figure 1. Domain region of interest

Table 1. Input features used for training the network.

\begin{tabular}{ll}
\hline Input Features & Feature Expressions \\
\hline Pressure gradient & $\frac{\partial\langle p\rangle}{\partial x}$ \\
Momentum convection & $\frac{\partial\langle p\rangle}{\partial y}$ \\
& $\frac{\partial\langle p\rangle\langle u\rangle\langle u\rangle}{\partial x}$ \\
& $\frac{\partial\langle p\rangle\langle u\rangle\langle v\rangle}{\partial y}$ \\
& $\frac{\partial\langle p\rangle\langle v\rangle\langle v\rangle}{\partial y}$ \\
Energy convection & $\frac{\partial\langle p\rangle\langle T\rangle\langle u\rangle}{\partial x}$ \\
Total heat flux & $\frac{\partial\langle p\rangle\langle T\rangle\langle v\rangle}{\partial y}$ \\
Velocity inlet & $q_{\text {total }}$ \\
Pressure inlet & $U_{\text {inlet }}$ \\
Temperature inlet & $p_{\text {inlet }}$ \\
Ambient pressure & $T_{\text {inlet }}$ \\
Fluid and gas viscosity & $p_{\text {amb }}$ \\
Non-dimensional x and y axis & $\mu_{l} \mu_{g}$ \\
& $x^{*}, y^{*}$
\end{tabular}

Table 2. Output features (quantities of interest).

\begin{tabular}{ll}
\hline Output Features & Feature Expressions \\
\hline Wall Temperature & $T_{\text {wall }}$ \\
Void Fraction & $\alpha$ \\
& \\
\hline
\end{tabular}

heat flux is applied, domain region of interest (ROI) is selected for data extraction. The number of cells in the ROI is $321 \times 26$ resulting in 8,346 data points for each case. The domain axis ( $\mathrm{x}$ and $\mathrm{y}$ ) is further converted into a nondimensional number so that the model is not constrained to learn based on the height of the channel and applicable for other channel lengths. In total 106 simulations have been performed for inlet velocity ranging from $0.05 \mathrm{~ms}^{-1}$ to $0.2 \mathrm{~ms}^{-1}$ and heat flux ranging from $1000 \mathrm{Wm}^{-2}$ to $40000 \mathrm{Wm}^{-2}$. 100 cases are used for training and validation, further, the data are split into $80 \%$ percent for training and $20 \%$ for validation purpose. The remaining 6 cases are used for evaluating the model performance.

The selected feature inputs obtained from CFD simulations are shown in the Table1. The inputs are chosen based on their influence on the quantities of interest. The 
quantities of interest chosen in this work are void fraction which controls the pressure drop and the flow regimes, and wall temperature which is relevant for cooling. The output of the deep neural network model is shown in the Table 2. Before feeding the data into the network, the input features and output features are normalized between 0 and 1 . This way the ML algorithms can learn better since the scale of data is very sparse. It is also worth mentioning that the data are randomly shuffled with a random instance of 8000 data points for every reshuffle. Shuffling the data ensures low variance and avoids the risk of creating batches that are not representative of the overall dataset while training the model.

\section{Deep Neural Network Architecture}

DNN is a type of Artificial Neural Network, where the number of hidden layers is more than one layer. The first and last layer serve as input and output layers similar to that of an ANN. The DNN architecture used in this work is shown in Figure 2. The network is trained using the backpropagation technique, meaning there are two phases involved in training the network. The first phase is called the forward phase. Here the signals from the input layer (training data) are propagated along the hidden layers with series of non-linear transformations controlled by weights and biases, followed by a nonlinear activation function until the output layer is reached.

$$
\begin{gathered}
h_{1}=\quad g\left(W_{1}^{T} x+b_{1}\right) \\
\cdot . \\
h_{5}=g\left(W_{5}^{T} h_{4}+b_{5}\right) \\
\hat{y}=g\left(W_{6}^{T} h_{5}+b_{6}\right) \\
g(x)= \begin{cases}0 & \text { for } x<0 \\
x & \text { for } x \geq 0\end{cases}
\end{gathered}
$$

The activation function $g(x)$ used in this work is rectified linear units (ReLU), where $h$ stands for hidden layers, $W$ is the weights of each layers, and $b$ is biases of the network.

A loss function is defined while training the network to measure the error between the predicted value $\hat{y}$ and the target value $y$. For this work, the mean squared error (MSE) is used as the loss function to compute the error then the error gradient is used to compute the new weights and biases of the neurons. Based on the computed gradients, the weights and biases are updated using Adaptive Moment Estimation (Adam) (Kingma and Ba, 2014) optimization technique in a backward direction with a learning rate of $\mathrm{Lr}=1 e^{-4}$. Hence, the second phase is called the backward phase.

The following measures have been taken to avoid overfitting while training the network. First and foremost $L_{2}$ regularization term is introduced in the loss function. Secondly, callback functions are defined to save only the best

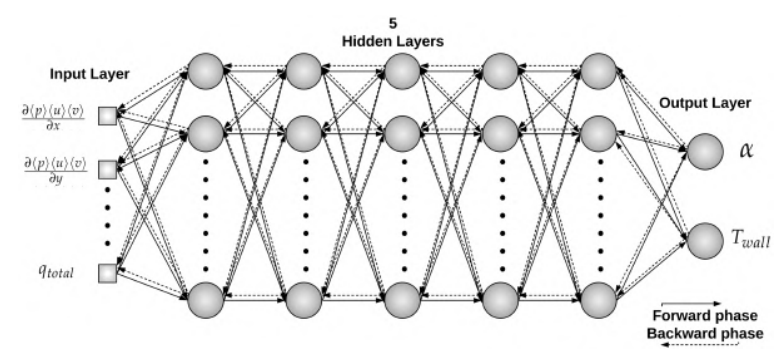

Figure 2. Architecture of the 5 hidden layer DNN.

weights of the network, early stopping of the training if the validation loss does not improve in the next epoch. Epoch is a term used when training a ML model, and it indicates the number of passes of the entire training dataset the ML algorithm has completed.

$$
\begin{array}{r}
L_{\text {loss }}=\operatorname{MSE}(y, \hat{y})+\lambda \sum_{i=1}^{N} w_{i}^{2} \\
L_{2} \text { norm }
\end{array}
$$

Where $\lambda$ is a positive hyperparameter that influences the regularization term, $N$ is the total number of data. While training the network other metrics such as, RMSE and coefficient of determination $\left(R^{2}\right)$ along with loss MSE function were defined to measure the accuracy of the model. Figure 3 shows the training RMSE and validation RMSE of the model trained for 500 epochs.

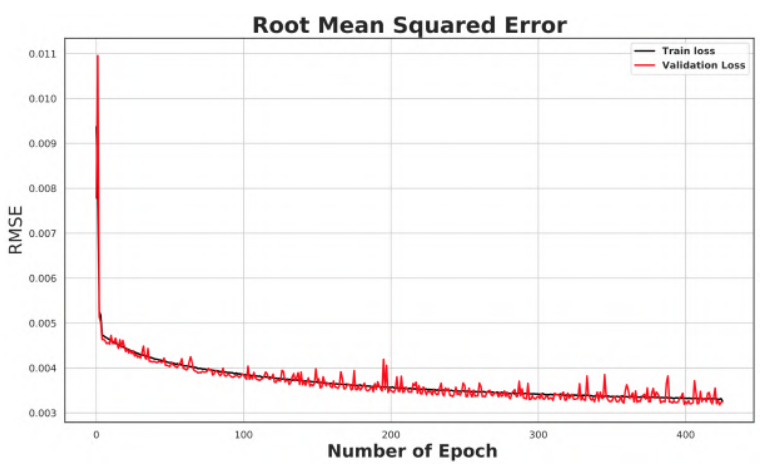

Figure 3. Training and validation RMSE error for 500 epochs.

In this work, the open source deep learning library Tensorflow 1.14 from google is used for constructing the model. The model is trained using GPU which allowed faster training compared to CPU.

\section{Results and Discussion}

\subsection{Validation dataset}

Before validation or evaluation of the model, the best weights of the model saved by the callback functions during training are first loaded. This confirms that the model weights used for validation and evaluation are the best one, and it is not an overfitted model. The errors in temperature, and void fraction values predicted using the DNN 


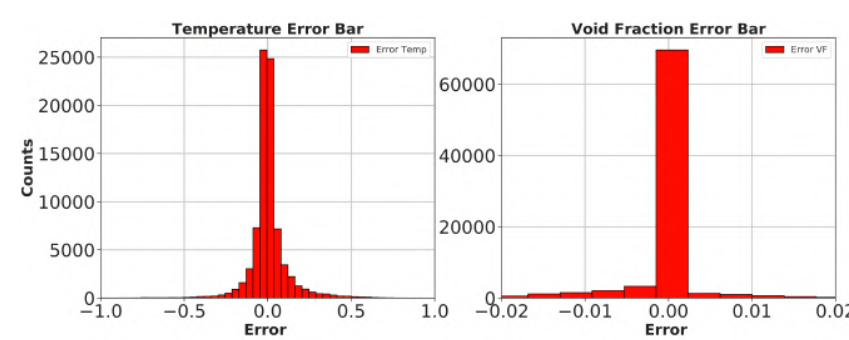

Figure 4. Predicted error on validation dataset for DNN model.

model from that of CFD values is illustrated in Figure 4. It is seen that the error lies around zero value for both the fields. Looking at the number of counts it can be interpreted that the DNN model slightly under predicts for the void fraction, since there are more counts on the negative side.

To get more insight of the model and its robustness, regression chart with relative error \% is shown in Figure 5. The relative error here is defined as: (Predicted value $_{-}$ $\left.C F D_{\text {value }}\right) * 100 / C F D_{\text {value }}$. The solid $45^{\circ}$ black line is the true line where DNN predicted values would perfectly match the CFD results. The blue dashed line stands for relative percentage error, the relative error is different for temperature and void fraction due to differences in scale. It can be further observed from the figure that the relative error for predicted temperature is under $0.5 \%$ error, which clearly shows that the DNN model can capture the temperature variation well. Whereas, in the case of the validated void fraction, the relative error is about $5 \%$, and it is seen that there are few outliers near-zero void fraction. The outliers present in the plots are statistically insignificant when compared to the number of data points (83460) present in the plot. The quantitative values of the DNN model on the
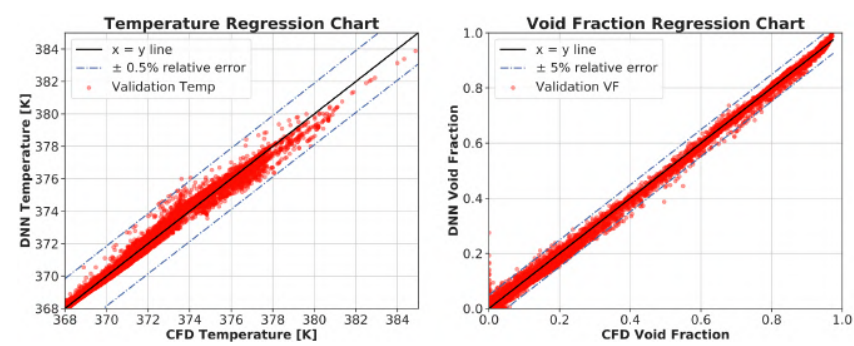

Figure 5. Comparison of CFD value and DNN value for the validation dataset.

validation dataset is presented in Table 3. From the table, it can be depicted that the model fits very well with the validation data and has a very low value of RMSE.

\subsection{Test dataset}

\subsubsection{Interpolation datasets}

Interpolation dataset here means that the chosen $\mathrm{Q}=$ $15000 \mathrm{Wm}^{-2}$ at velocity inlet $\mathrm{u}=0.05 \mathrm{~ms}^{-1}$ lies between the range of training data, but this data were not used for training nor validation. Once the model has been
Table 3. Statistics table of the validation dataset. Val: Validation, VF: Void Fraction, Temp: Temperature

\begin{tabular}{llclc}
\hline \multirow{2}{*}{ Dataset } & \multicolumn{2}{c}{$R^{2}$} & \multicolumn{2}{c}{ RMSE } \\
& VF & Temp & VF & Temp \\
\hline Val & 0.998 & 0.995 & 0.0059 & 0.134 \\
\hline \hline
\end{tabular}

validated, the unseen test data are used to evaluate the model. Figure 6 shows the temperature field obtained from a CFD result, the temperature field predicted using the DNN model, and the relative percentage error between them. It is noticeable from the figure that the error ranges between $\pm 0.3 \%$, which implies the DNN model can capture the complex nature, and the model could be used for predicting unseen cases. However, the model slightly over predicts the temperature field near the wall of the channel, and under predict around the center of the channel. To understand furthermore and to closely observe the performance of the model in near-wall region Figure 7 is plotted, which shows the trend of void fraction and wall temperature along the channel. From the figure it can be further noted that the DNN model is capable of predicting the subcooled boiling regimes closely to that of CFD values.

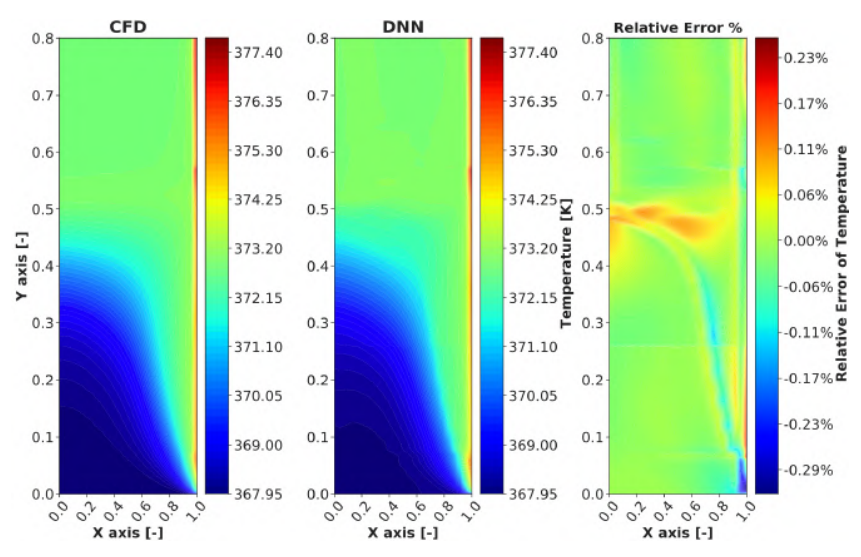

Figure 6. Temperature field for CFD, DNN and relative error \% for $\mathrm{Q}=15,000 \mathrm{Wm}^{-2}$ at $\mathrm{u}=0.05 \mathrm{~ms}^{-1}$.
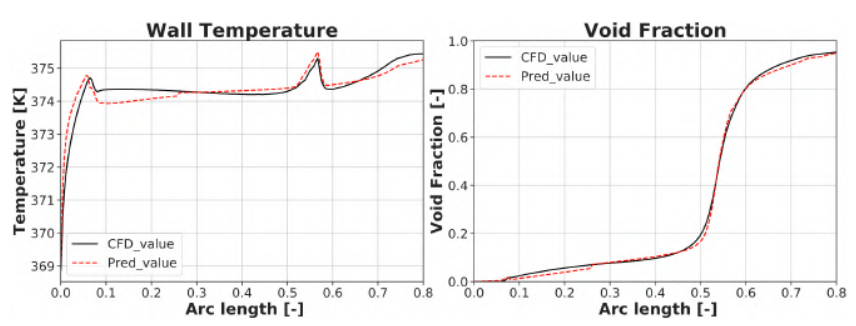

Figure 7. CFD, DNN wall void fraction and wall temperature for $\mathrm{Q}=15,000 \mathrm{Wm}^{-2}$ at $\mathrm{u}=0.05 \mathrm{~ms}^{-1}$. Arc length: The non-dimensional length along the y-axis near the wall of the minichannel.

The void fraction field in the channel obtained from CFD and predicted results from DNN is presented in Fig- 

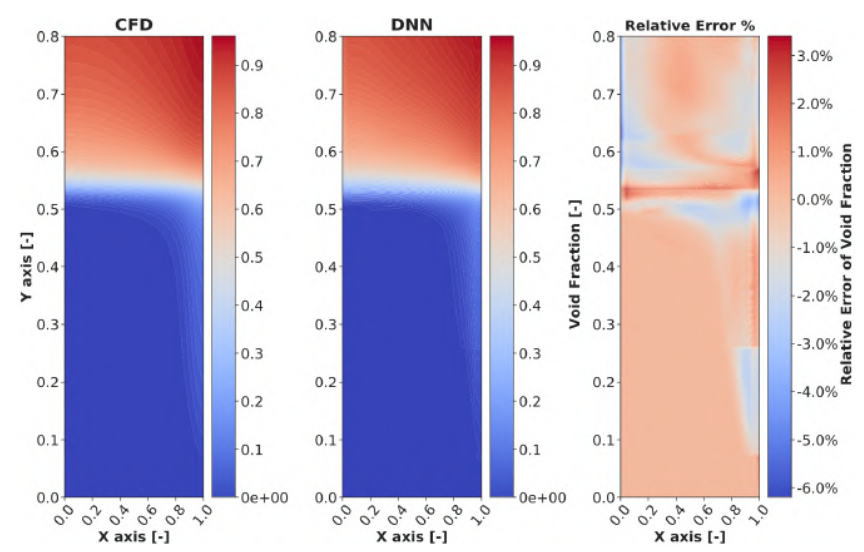

Figure 8. Void fraction field for CFD, DNN and relative error $\%$ for $\mathrm{Q}=15,000 \mathrm{Wm}^{-2}$ at $\mathrm{u}=0.05 \mathrm{~ms}^{-1}$.

ure 8. Here it can be seen that the relative error ranges from $3.2 \%$ to $-6 \%$, and this error is mostly concentrated near the non-dimensional arc length of 0.5 where the void fraction suddenly starts to increase. This sudden change in void fraction can be observed in Figure 7, and it can be observed that temperature is closely related to this phenomenon. One explanation could be due to the presence of a hotspot, caused by the formation of a bigger bubble or a large number of bubbles near the wall resulting in a sudden increase in void fraction and temperature.

Figure 9 shows how well the model fits on the interpolation test data. From the plot, it can be spotted that the trend of error is similar to that of the validation dataset, but the number of outliers is lower because the test data is 10 times less than that of validation data. Again from the temperature regression chart, it is noticeable that there is an offset in predicted temperature around $374 \mathrm{~K}$, but the relative error is still under $0.3 \%$. With the above-presented results and from Table4, it is possible to conclude that the DNN model can predict well for interpolation cases.
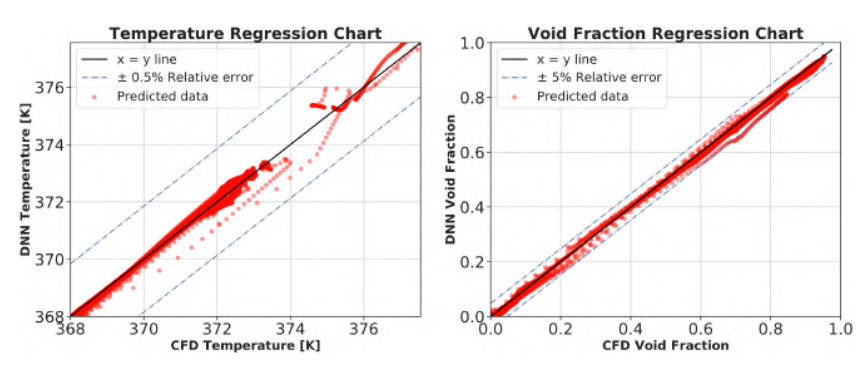

Figure 9. CFD, DNN regression chart of void fraction and temperature for $\mathrm{Q}=15,000 \mathrm{Wm}^{-2}$ at $\mathrm{u}=0.05 \mathrm{~ms}^{-1}$.

\subsubsection{Extrapolation dataset}

The dataset used for extrapolation is an extreme case, meaning this data is far away from any data that is used during training of the model. It is to be noted that the extrapolation data sets are already available through CFD calculation but it is not used for training the DNN. The maximum heat flux and velocity used during training
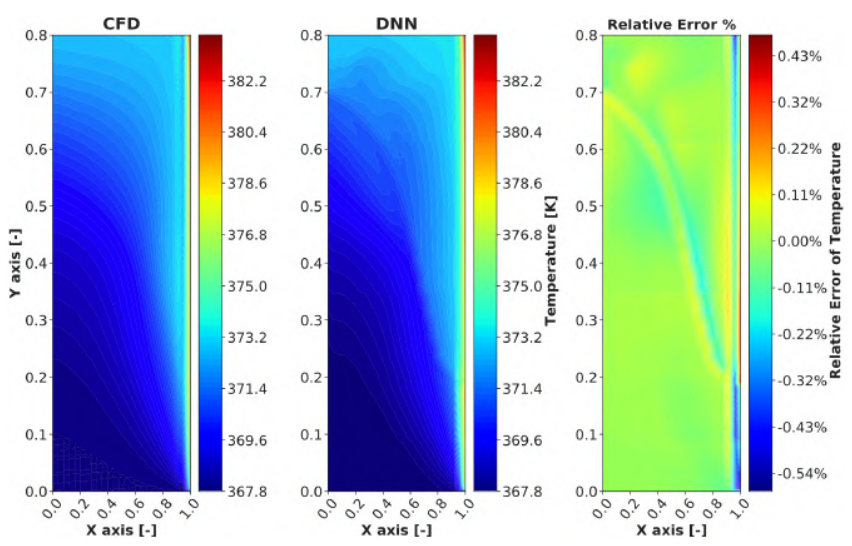

Figure 10. Temperature field for CFD, DNN and relative error $\%$ for $\mathrm{Q}=40,000 \mathrm{Wm}^{-2}$ at $\mathrm{u}=0.2 \mathrm{~ms}^{-1}$.

ranges from $\mathrm{Q}=1000$ to $29,000 \mathrm{Wm}^{-2}$ and $\mathrm{u}=0.05$ to 0.2 $m s^{-1}$, while the data used for extrapolation have a heat flux value of $\mathrm{Q}=40,000 \mathrm{Wm}^{-2}$ at $\mathrm{u}=0.2 \mathrm{~ms}^{-1}$. The motive was to see if the DNN model has learned sufficiently the underlying physics of subcooled boiling from the data provided during training, and hence reproduce the physics on unseen cases away from the training region.

The temperature field obtained from CFD data and the predicted value by the DNN model is shown in Figure 10. From the figure it can be observed that the DNN model follows the same pattern of temperature distribution along the channel. The next plot shows how far is the predicted value from the CFD value, and it is worth noticing that the relative error is still under $\pm 0.5 \%$ error. The possible explanation for this behavior could be that the DNN model has the capability of learning from the local features and intrinsic pattern present in the training data, and it can extrapolate the data points to explain the physical process of subcooled boiling temperature.
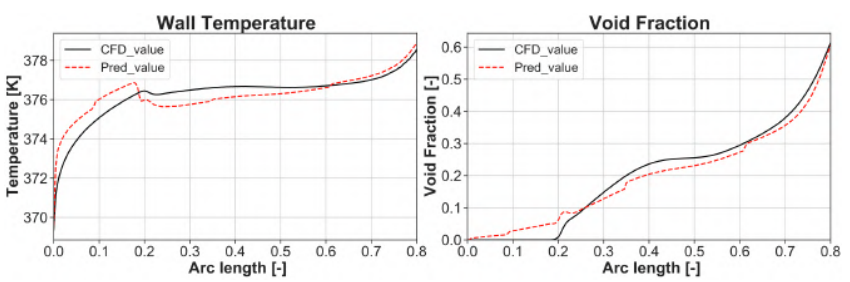

Figure 11. CFD, DNN wall void fraction and wall temperature for $\mathrm{Q}=40,000 \mathrm{Wm}^{-2}$ at $\mathrm{u}=0.2 \mathrm{~ms}^{-1}$.

The void fraction and the wall temperature near the wall region are illustrated in Figure 11. From the plot, it can be noted that the DNN model over predicts until the arc length of 0.24 and it starts to under predicts until 0.75 arc length then follows the pattern of CFD data. From this, it is possible to conclude that the DNN model finds it difficult to capture the void fraction when its value is equal to zero. The temperature profile near the wall, however, follows a good trend compared to CFD data. 
Table 4. Performance of the DNN model on test dataset, VF: Void Fraction, Temp: Temperature, Inter: Interpolation, Extra: Extrapolation. *: Results presented in the paper.

\begin{tabular}{|c|c|c|c|c|c|c|c|c|}
\hline \multirow[t]{2}{*}{ Cases } & \multirow{2}{*}{$\underset{m s^{-1}}{\mathrm{U}}$} & \multirow{2}{*}{$\begin{array}{c}\mathrm{Q} \\
W m^{-2}\end{array}$} & \multicolumn{2}{|c|}{ RMSEP } & \multicolumn{2}{|c|}{$R^{2}$} & \multicolumn{2}{|c|}{ Error(\%) } \\
\hline & & & VF & Temp & VF & Temp & VF & Temp \\
\hline \multirow{2}{*}{ Inter* } & 0.05 & 15000 & 0.0088 & 0.13 & 0.999 & 0.995 & 3.64 & 0.31 \\
\hline & & & & & & & -6.09 & -0.26 \\
\hline \multirow{2}{*}{ Inter } & 0.075 & 17500 & 0.0066 & 0.129 & 0.999 & 0.996 & 4.3 & 0.26 \\
\hline & & & & & & & -4.5 & -0.36 \\
\hline \multirow{2}{*}{ Extra } & 0.1 & 30000 & 0.011 & 0.23 & 0.998 & 0.98 & 9.48 & 0.617 \\
\hline & & & & & & & -8.33 & -0.303 \\
\hline \multirow{2}{*}{ Inter } & 0.15 & 19000 & 0.0025 & 0.08 & 0.96 & 0.99 & 2.4 & 0.121 \\
\hline & & & & & & & -1.4 & -0.27 \\
\hline \multirow{2}{*}{ Extra } & 0.15 & 30000 & 0.0084 & 0.196 & 0.98 & 0.99 & 7.07 & 0.4 \\
\hline & & & & & & & -9.29 & -0.414 \\
\hline \multirow{2}{*}{ Extra* } & 0.2 & 40000 & 0.013 & 0.278 & 0.982 & 0.987 & 10.8 & 0.58 \\
\hline & & & & & & & -10.5 & -0.47 \\
\hline
\end{tabular}
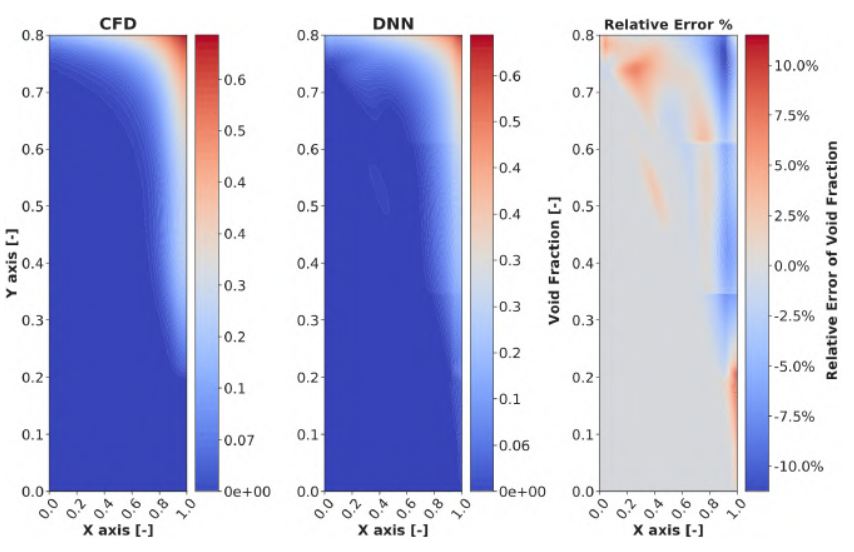

Figure 12. Void fraction field for CFD, DNN and relative error $\% \mathrm{Q}=40,000 \mathrm{Wm}^{-2}$ at $\mathrm{u}=0.2 \mathrm{~ms}^{-1}$.

The CFD domain and the domain predicted using the DNN model is indicated in Figure 12. From the relative error plot it is noticeable that the maximum relative error is in the range of $\pm 10.5 \%$. These errors are mostly concentrated near the wall, and the upper region of the channel. The possible explanation for such a behavior may be due to the lack of data for such high heat flux value in the training data and the physical nature of the problem in these region is difficult to predict resulting in poor prediction. Nevertheless, the DNN model still follows the trend of CFD void fraction value, and it can predict the whole domain under $10 \%$ relative error. Figure 13 shows how well the model fits the extrapolation data, for void fraction around $90 \%$ of the data lies between $\pm 5 \%$ relative error to that of the true line (solid black line) and remaining $10 \%$ of the data points are the sparse one which inflicts the maximum error in the model. Looking at temperature data distribution, there are some non-linear data points around $376 \mathrm{~K}$, which accounts for $0.5 \%$ relative error in the model.
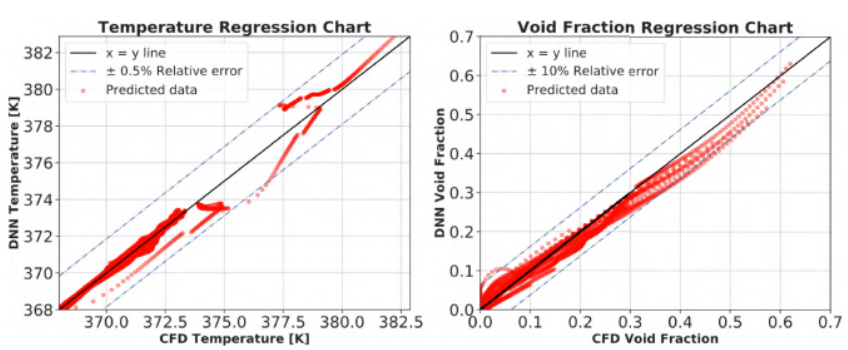

Figure 13. CFD, DNN regression chart of void fraction and temperature for $\mathrm{Q}=40,000 \mathrm{Wm}^{-2}$ at $\mathrm{u}=0.2 \mathrm{~ms}^{-1}$.

The model performance for all the test data is presented in Table 4. The test data consist of interpolation and extrapolation dataset. It is noticeable that the model is evaluated, on quite a variety of datasets. This provides a strong indication of how generalized the model used in this work is. It is worth noticing that the RMSEP values are well below 0.3 , and the coefficient of determination score is about 0.98 on average. This shows how well the model fits the data. From the presented results, it is possible to conclude that the model performs very well when predicting the temperature field in the domain with a maximum relative error of $0.6 \%$ for both interpolation and extrapolation datasets. However, the models still lacks good accuracy when it comes to predicting the void fraction field, especially near the wall for the extrapolation datasets. Nonetheless, the model can still follow the trend of CFD values and predict well for the interpolation datasets, and for extrapolation datasets that are not far away from the values used for training. 


\section{Conclusion and Future Work}

In this work, a supervised deep neural network model based on the backpropagation technique is implemented to study the subcooled boiling heat transfer. The training data are obtained from CFD simulations from varying heat fluxes and inlet velocities. In total 106 cases were simulated, out of which 80 cases were used for training the network, 20 cases for validation purposes, and the remaining 6 cases for evaluating the model. After training, the performance of the DNN model was validated using the validation dataset, and then the model performance was evaluated using test datasets. The model showed good level of accuracy while predicting temperature and void fraction field for both interpolation and extrapolation dataset. In the case of extreme extrapolation, the model performance deteriorated while predicting the void fraction field with a maximum error of $10.8 \%$. Nonetheless, the model showed an exceptional level of accuracy while predicting the temperature field with a maximum error of $0.6 \%$. From the results obtained in this work, it is demonstrated that the DNN model is capable of capturing the subcooled boiling pattern, and it is applicable for interpolation and extrapolation data extraction of the quantities of interest. Moreover, the DNN model trained on the local flow features has acceptable generalization property, and it can be used as a promising tool to help improve the predictive speed of MCFD solvers. Therefore, the future work will focus on improving the predictive nature of the DNN model for void fraction field, and for quantifying the uncertainty present in the DNN model.

\section{Acknowledgements}

The authors would like to thank Mr. Achref Rabhi for carrying out the CFD simulations and providing the data needed to conduct this research. This research was conducted at Mälardalen University.

\section{References}

Erdem Alic, Mehmet Das, and Onder Kaska. Heat flux estimation at pool boiling processes with computational intelligence methods. Processes, 7(5):293, 2019.

A Ansari, SSH Boosari, and SD Mohaghegh. Successful implementation of artificial intelligence and machine learning in multiphase flow: Two case studies of cfd problems. J Pet Environ Biotechnol, 11:401, 2020.

RJ Benjamin and AR Balakrishnan. Nucleation site density in pool boiling of saturated pure liquids: effect of surface microroughness and surface and liquid physical properties. Experimental Thermal and Fluid Science, 15(1):32-42, 1997.

Giorgio Besagni, Fabio Inzoli, and Thomas Ziegenhein. Twophase bubble columns: A comprehensive review. ChemEngineering, 2(2):13, 2018.

Caleb S Brooks and Takashi Hibiki. Wall nucleation modeling in subcooled boiling flow. International Journal of Heat and Mass Transfer, 86:183-196, 2015.
JFH Buist, Benjamin Sanderse, Yous van Halder, Barry Koren, and GertJan van Heijst. Machine Learning for Closure Models in Multiphase-Flow Applications. PhD thesis, Master's thesis, Eindhoven University of Technology, 2019.

SCP Cheung, S Vahaji, GH Yeoh, and JY Tu. Modeling subcooled flow boiling in vertical channels at low pressures-part 1: Assessment of empirical correlations. International Journal of Heat and Mass Transfer, 75:736-753, 2014.

Gustavo M Hobold and Alexandre K da Silva. Visualizationbased nucleate boiling heat flux quantification using machine learning. International Journal of Heat and Mass Transfer, 134:511-520, 2019.

Mamoru Ishii. Thermo-fluid dynamic theory of two-phase flow. STIA, 75:29657, 1975.

Hugo A Jakobsen, Håvard Lindborg, and Carlos A Dorao. Modeling of bubble column reactors: progress and limitations. Industrial \& engineering chemistry research, 44(14):51075151, 2005.

Satish G Kandlikar. Heat transfer characteristics in partial boiling, fully developed boiling, and significant void flow regions of subcooled flow boiling. 1998.

Diederik P Kingma and Jimmy Ba. Adam: A method for stochastic optimization. arXiv preprint arXiv:1412.6980, 2014.

Harsha Kumar and Gnanasekaran Nagarajan. A synergistic combination of asymptotic computational fluid dynamics and ANN for the estimation of unknown heat flux from fin heat transfer. Alexandria Engineering Journal, 57(2):555-564, 2018.

$\mathrm{N}$ Kurul. On the modeling of multidimensional effects in boiling channels. ANS. Proc. National Heat Transfer Con. Minneapolis, Minnesota, USA, 1991, 1991.

Richard T Lahey Jr. The simulation of multidimensional multiphase flows. Nuclear Engineering and Design, 235(10-12): 1043-1060, 2005.

Ming Ma, Jiacai Lu, and Gretar Tryggvason. Using statistical learning to close two-fluid multiphase flow equations for a simple bubbly system. Physics of Fluids, 27(9):092101, 2015.

Shah Mohammed and Mohammed Shah. A general correlation for heat transfer during subcooled boiling in pipes and annuli. 1977.

HC Ünal. Maximum bubble diameter, maximum bubble-growth time and bubble-growth rate during the subcooled nucleate flow boiling of water up to $17.7 \mathrm{mn} / \mathrm{m} 2$. International Journal of Heat and Mass Transfer, 19(6):643-649, 1976.

Chih-Ping Yin, Yi-Yie Yan, Tsing-Fa Lin, and Bing-Chwen Yang. Subcooled flow boiling heat transfer of r-134a and bubble characteristics in a horizontal annular duct. International journal of heat and mass transfer, 43(11):1885-1896, 2000. 